\title{
Evidence for a spectral turnover in the broadband gamma-ray emission from SNR Puppis A revealed by H.E.S.S. observations
}

\author{
I. Oya* \\ DESY, Zeuthen, Germany \\ E-mail: igor.oya.vallejo@desy.de

\section{Fernandez} \\ Laboratoire Univers et Particules de Montpellier, Université Montpellier 2, CNRS/IN2P3, \\ Montpellier, France

\section{Renaud} \\ Laboratoire Univers et Particules de Montpellier, Université Montpellier 2, CNRS/IN2P3, \\ Montpellier, France
}

\section{R.C.G. Chaves}

Laboratoire Univers et Particules de Montpellier, Université Montpellier 2, CNRS/IN2P3, Montpellier, France

\section{S. Gabici}

APC, AstroParticule et Cosmologie, Université Paris Diderot, CNRS/IN2P3, CEA/Irfu, Observatoire de Paris, Sorbonne Paris Cité, Paris, France

\section{For the H.E.S.S. collaboration}

The 4 kyr-old supernova remnant (SNR) Puppis A shows strong evidence of interaction between the forward shock and a molecular cloud. The results from Fermi-LAT indicate extended highenergy gamma-ray emission with a $0.2-100 \mathrm{GeV}$ spectrum that does not significantly deviate from a power law, in contrast to most of the GeV-bright SNRs known to be interacting with molecular clouds. In order to characterize the position of a spectral feature at higher energies, very-highenergy $(\mathrm{E}>0.1 \mathrm{TeV})$ gamma-ray observations of Puppis A were carried out with the High Energy Stereoscopic System (H.E.S.S.) telescope array. The analysis of the H.E.S.S. data did not reveal any significant emission towards Puppis A. The upper limits on the differential photon flux imply that its broadband gamma-ray spectrum must exhibit a spectral break, estimated to be below 280 $\mathrm{GeV}$, or alternatively a cutoff below $450 \mathrm{GeV}$ or $280 \mathrm{GeV}$ when assuming exponential or subexponential cutoff in the power law spectrum, respectively. These results provide insights into our understanding of the processes accelerating particles in the shock front of Puppis A. The details of this work have been published in [1].

The 34th International Cosmic Ray Conference,

30 July- 6 August, 2015

The Hague, The Netherlands

\footnotetext{
* Speaker.
} 


\section{References}

[1] Abramowski, A., Aharonian, F., Ait Benkhali, F., et al. H.E.S.S. reveals a lack of TeV emission from the supernova remnant Puppis A, A\&A, 575 (2015), A81. 